\title{
The effect of strain of rats on the acquisition of discriminated avoidance response
}

H. M. B. HURWITZ AND PAUL V. DILLOW

UNIVERSITY OF TENNESSEE

Twenty naive female hooded rats were trained in a leverpressing apparatus under a discriminated avoidance procedure in a single $4 \mathrm{hr}$. session. Ten Ss were derived from a laboratory where brother-sister mating had been enforced for 45 generations over 19 years (Group G); 10 Ss were derived from a laboratory where random within-colony breeding had been used for 37 years (Group MRC). The groups differed in terms of variance between Ss but not in terms of the mean rate of acquiring the avoidance response, the pure strain showing a significantly smaller between-S variance. $(p<.01)$

It is well known that under an avoidance procedure (1) many animals fail to reach the required criterion of acquisition, even when a liberal criterion prevails; (2) different species produce different results under identical circumstances, e.g., cats, but not rats, readily learn to lever press in response to the warning signal; (3) measure of learning, like latency of response to the signal, probability of response in the signal period, percent avoidance as a function of trials, typically show a very large between subject variability which may mask the effect of an independent variable. The present study examines the possibility that a major contribution to the size of the within-group variance in a groupdesigned experiment may be attributable to a genetic factor, specifically, to the strain of animal used. Subjects

The Ss were 20 experimentally naive, female hooded rats. They were three months old at the time of experimentation. Ten Ss were purchased from the closed colony maintained by the animal laboratories of the Medical Research Council, London. These laboratories employ a random within-colony mating procedure on a stock of hooded rats supplied in 1929 by Imperial Chemical Industry Laboratories. Ten Ss were donated by Glaxo Laboratories, London. These laboratories maintain a brother-sister mating procedure on a strain developed by Virol, Ltd., London in 1947. The Ss used in the experiment were drawn from the 45 th generation so bred. Apparatus

The basic apparatus was an all-metal chamber, $12 \mathrm{in.}$ long, $6 \mathrm{in}$. wide, and $9 \mathrm{in}$. high. The floor of the chamber consisted of five $1 / 4$ in. bars through which shock could be delivered to the Ss' feet. A stainless steel lever measuring 2 in. wide, .75 in. long and . 36 in. thick protruded through one of the walls of the chamber $2.5 \mathrm{in}$. above floor level. This operated a microswitch when pressed downward with a force of $5 \mathrm{gm}$. Shock was applied to the grid via a scrambler. Two panel lights served as signals: one was mounted above the lever, the other at the far side of the chamber. A .8 constant current electric shock was used as the aversive stimulus.

\section{Procedure}

Each $\mathrm{S}$ was given a single training session which lasted for $4 \mathrm{hr}$. Shortly after the animal was placed in the chamber, the signal-light was presented and unless the lever was pressed within 9 sec., the first of a series of brief shocks ( $0.5 \mathrm{sec}$. on; $5 \mathrm{sec}$. off) was given. The signal and the series of shocks were terminated by a lever press. A press during the brief shock-on period had no effect, i.e., shock could not be escaped (Hurwitz, 1964). The next presentation of the signal and the series of shocks was scheduled 18 sec. after such a response irrespective of whether the response had averted or had terminated the shock-series.

Results

The percent avoidance and the scores of the number of shocks received by each $S$ in each of the two groups are shown in Figs. 1 and 2 respectively. A statistical analysis, designed to test the hypothesis that the variance of the two groups was equal, was undertaken on the number of shocks received during each of the $4 \mathrm{hr}$. of the training session and on the percent avoidance scores (i.e., the number of occasions that a response was effective in averting the series of shocks was expressed as a percentage of the total number of responses which had been effective in either averting the shock series or in terminating it). This analysis showed that the variance in the number of shocks received differed significantly at the $p=.001$ level of significance at the second, third and fourth hour (Fig. 1; $\mathrm{F}=14.4,145.6$ and 18.9, respectively; Group MRC $>$ Group G). The

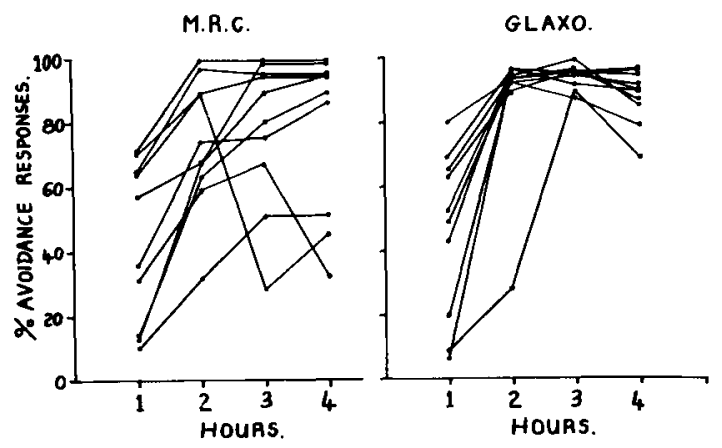

Fig. 1. Percent avoidance responses under a discriminated avoidance procedure expressed as a function of the time period of training for animals obtained from Medical Research Council and Glaxo Laboratories respectively. 


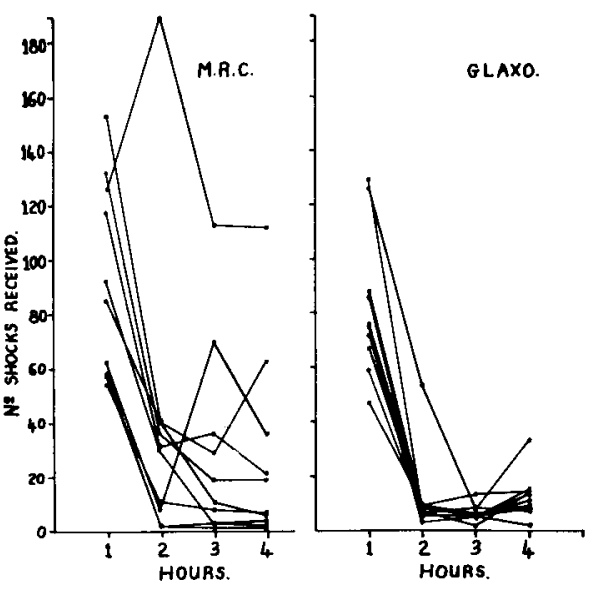

Fig. 2. Number of shocks received under a discriminated avoidance procedure expressed as a function of the time period of training for animals obtained from Medical Research Council and Glaxo Laboratories respectively.

variance in the percent avoidance showed a similar trend, significant differences being found in the third and fourth hour at the $p=.01$ level of significance $(F=$ 41.5 and 9.7 respectively) (Fig. 2).

An analysis of variance to test the hypothesis that hourly means of each group were equal was excluded because of the non-homogeneity of scores, so that ttests appeared appropriate to assess the significance of the difference between hourly means. Because of the differences between variances at each hour, Welch's formula (as outlined by Winer, 1962) had to be used to compute degrees of freedom. This showed that the means for the number of shocks differed only on the third hour, $p=.05, t=2.7$. No significant difference between scores for percent avoidance was found.

\section{Discussion}

The results clearly demonstrate that the two genetically different strains performed very differently under the discriminated avoidance procedure. Ss derived from a brother-sister bred colony (Group $\mathrm{G}$ ) were relatively uniform in their behavioral adjustment to the training situation in contrast to Ss derived from an inbred colony where a random breeding procedure is practiced. A casual inspection of the graphs may lead to the conclusion that Group $G$ acquired the avoidance significantly faster than Group MRC, but this is not supported by our statistical analysis.

We have surveyed a large number of currentand past studies of discriminated avoidance learning to find out something about the strain of Ss used. In almost all cases Ss were derived from commercial suppliers who at best adopt a random within-colony breeding schedule.
This may account for the large within-group variance reported. Exceptions are studies in which strain difference entered as a systematic variable (Myers, 1959), or where, by chance, a pure strain was available (e.g., Hurwitz, 1964).

Our results should not be taken to support the generalization that pure strains necessarily yield behavioral measures with smaller variances than when animals of heterozygotic stock are used. Nor do the results support the proposition that pure strains are likely to be faster learners of avoidance behavior. Behavioral variability, especially as detected under a procedure involving aversive stimulation, could equally well be a characteristic of a relatively pure stock but of a different genetic constitution than the strain used in our study. Thus our results are not in conflict with those recently reported by Collins (1964) in which the avoidance learning of five pure mice strains were compared to the learning rate of hybrid ss derived from interbreeding the pure strains. It was found that the hybrids were markedly superior to the parent Ss in acquiring the discriminated avoidance response of pure strains. Collins believes that since avoidance learning may be of significant adaptive value to the species, the superiority of the hybrids supports the view of hybrid viability. Presumably this generalization applies only to those cases where the pure strain does not already possess the characteristic under investigation to an advanced degree, but where such a characteristic is either normally distributed in the population or perhaps exists to a subnormal degree.

Because the experiment has demonstrated the importance of the strain of animals in determining behavioral variability it becomes advisable, if not mandatory, for experimenters who propose to use rate of learning an avoidance response as their principal dependent variable to run Ss derived from a strain known to produce small inter-subject variability, since this will increase their chances of detecting subtle, though important factors determining the acquisition and subsequent maintenance of avoidance behavior.

\section{References}

Collins, Robert L. Inheritance of avoidance conditioning in mice: A diallel study. Science, 1964, 143, 1188-1190.

Hurwitz, H. M. B. Method for discriminative avoidance training. Science, 1964, 145, 1070-1071.

Hurwitz, H. M. B. The effect of constant current shock intensities on the acquisition of a discriminated avoidance response. Psychon. Sci., 1966, 5(3), 109-110.

Myers, Arlo K. Avoidance learning as a function of several training conditions and strain differences in rats. J. comp. physiol. Psychol., 1959, 52, 381-386.

Winer, B. J. Statistical principles in experimental design. New York: McGraw-Hill, 1962. 